\title{
Digitalisierung messbar machen
}

\section{Ein soziotechnisches KPI-Modell für die digitale Transformation}

\author{
Felix Krol, Birgit von See und Wolfgang Kersten, Technische Universität Hamburg
}

Measuring Digitalization - A Sociotechnical KPI Model for the Digital Transformation

A successful digital transformation for attaining Industry 4.0, is a crucial success criterion for many companies today. The ongoing global COVID-19 pandemic has shown the need for digitalization in companies and has further accelerated this development. However, these times, companies are confronted with an uncertain order and profit situation. Thus, they need to allocate their investments purposefully. Evaluating the digital maturity by using a profound indicator system is therefore a sound basis for decision making. This paper develops such a sociotechnical KPI model along the dimensions "Strategy and Organizational Leadership"," "Digital Skills/Human Capital" as well as "Smart Process/Operations". In the future, this model can be used for determining the digital maturity and thus, it can be applied for allocating digitalization investments.

Keywords:

digital transformation, indicators, socio-technical system, industry 4.0

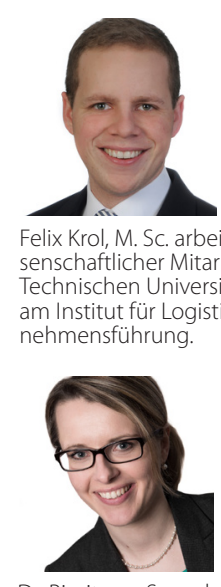

Dr. Birgit von See arbeitet als Postdoc und stellvertretende Oberingenieuund stellvertretende Oberingenieu-
rin an der Technischen Universität rin an der Technischen Universität
Hamburg am Institut für Logistik und Unternehmensführung.

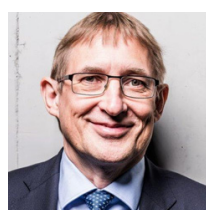

Prof. Dr. Dr. h. c. Wolfgang Kersten leitet an der Technischen Universität Hamburg das Institut für Logistik und Unternehmensführung.

logu@tuhh.de www.logu.tuhh.de

Eine erfolgreiche digitale Transformation hin zum Zielbild der Industrie 4.0 ist heute bereits für viele Unternehmen ein entscheidendes Wettbewerbskriterium. Die anhaltende globale COVID-19-Pandemie hat die Notwendigkeit der Digitalisierung in den Unternehmen deutlich gemacht und diese zusätzlich beschleunigt. Insbesondere in dieser Zeit sind die Unternehmen jedoch mit einer ungewissen Auftrags- und Ertragslage konfrontiert und müssen ihre Investitionsbudgets zielgerichtet einsetzen. Als Entscheidungsgrundlage bietet sich die Bestimmung der digitalen Reife mithilfe eines fundierten Kennzahlensystems an. Dieser Beitrag entwickelt ein solches soziotechnisches Digitalisierungs-KPI-Modell entlang der Dimensionen „Strategie und Führung“, „Digitalkompetenzen/Humankapital“ und „Intelligente Prozesse”. Das Modell kann zukünftig als Grundlage für die Bestimmung des digitalen Reifegrads und darauf aufbauend der Allokation von Digitalisierungs-Investitionen genutzt werden.

Dieser Beitrag fokussiert die Messbarkeit der fortschreitenden digitalen Transformation durch ein Kennzahlensystem. Durch die globale COVID-19-Pandemie hat sich die digitale Transformation, die das Zielbild der Industrie 4.0 verfolgt, seit dem 1. Halbjahr 2020 deutlich beschleunigt und wird für (produzierende) Unternehmen auch in den kommenden Jahren eine hohe wirtschaftliche Relevanz haben [1]. So bewerten Unternehmen in Logistik und Supply Chain Management die Digitalisierung von Geschäftsprozessen heute als den für sie relevantesten Trend [2]. Trotz der anhaltenden Corona-Krise sind die Sicherung von Innovations-/Wettbewerbsfähigkeit und die Gestaltung der Digitalisierung die wichtigsten Handlungsfelder für kleine und mittlere Unternehmen (KMU) [3].

Dennoch ist die Auftrags- und Ertragslage insbesondere für KMU derzeit mit hohen Unsicherheiten belastet. Akut hat für viele KMU die Sicherung der eigenen Liquidität oberste Priorität [3]. Die verbleibenden, geringen Investitionsbudgets für die Digitalisierung müssen mehr denn je zielgerichtet in die erfolgversprechendsten Geschäftsfelder und Technologien investiert werden.

Neben stark begrenzten Investitionsbudgets sind insbesondere fehlende Digitalisierungskompetenzen der Belegschaft ein wesentliches
Hemmnis für die mittelständische Digitalisierung [4]. Die Mehrheit der KMU verhält sich in der digitalen Transformation passiv und kann die Bedeutung von disruptiven Entwicklungen für das eigene Unternehmen nicht einschätzen [5]. Diese Unternehmen sind besonders durch das Risiko betroffen, aufgrund ihrer fehlenden digitalen Reife mittelfristig aus der Supply Chain auszuscheiden [6].

Diese Ursachen lassen die Schlussfolgerung $z u$, dass insbesondere KMU ein Messwerkzeug fehlt, mit dem sie fundiert den Status quo ihrer digitalen Reife bestimmen können. Durch die Bewertung der digitalen Reife kann anschließend eine zielgerichtete Priorisierung der nächsten Schritte sowie eine Allokation der damit verbundenen Investitionen vorgenommen werden. Dazu werden zunächst aussagekräftige Messgrößen benötigt, mit denen der Status quo der digitalen Transformation bestimmt werden kann. In diesem Beitrag wird für alle Messgrößen übergreifend der Begriff Key Performance Indikator (KPI) verwendet. Ob die jeweilige Messgröße als (Key) Performance Indikator definiert wird, muss vom anwendenden Unternehmen eigenständig und individuell festgelegt werden [7]. Vor dem Hintergrund der Mensch-Technik-Interaktion in der digitalisierten Arbeitswelt wird im Nachfolgenden ein soziotechnisches KPI-Modell entwickelt, das für die Entwicklung eines Digitalisierungs-Reifegradmodells genutzt werden kann. 


\section{Forschungsansatz}

Das entwickelte KPI-Modell basiert auf einem zweistufigen, literaturbasierten Forschungsansatz. In einem ersten Schritt wurden mittels einer Literatur- und Internetrecherche (Schneeballsystem) übergeordnete Themenfelder (insgesamt neun Dimensionen und 30 Subdimensionen) für das KPI-Modell identifiziert. Die strukturierte Auswahl ebendieser fußt auf der Analyse von 19 bestehenden Digitalisierungs-Reifegradmodellen aus sowohl wissenschaftlichen als auch praxisorientierten Beiträgen.

In einem darauffolgenden zweiten Schritt wurden dann mithilfe einer Schlagwortsuche insgesamt 965 KPIs identifiziert. Basis der Analyse bildete eine Kombination aus einer systematische Literaturrecherche nach Fink [8] sowie einer ergänzenden Recherche wissenschaftlicher und praxisorientierter Quellen. Der verwendete Suchstring sowieso das Vorgehen in der systematischen Literaturrecherche wird in Bild 1 ersichtlich. Mittels einer zweistufigen Evaluierung durch vier Wissenschaftler*innen wurden die Indikatoren ausgewählt, die zur Ermittlung des Status quo der digitalen Transformation von produzierenden Unternehmen genutzt werden können. Im Ergebnis wurden 488 Digitalisierungs-KPI in das Modell integriert, die in der Evaluation einen Konsens erzielten. Anschließend wurden sowohl den KPI als auch den, im ersten Schritt identifizierten, Subthemen Schlagwörter zugeordnet, wodurch eine automatisierte Zuordnung umgesetzt werden konnte. Es erfolgte eine abschließende Überprüfung, Anpassung sowie Konsolidierung zu aussagekräftigen und messbaren KPI.

Mit einer Fokussierung auf die Mensch-Technik-Interaktion werden in diesem Beitrag die drei soziotechnischen Themenfelder "Strategie und Führung", Digitalkompetenzen/Humankapital" sowie "Intelligente Prozesse" dargestellt. Das vorgestellte Ergebnis stellt einen Ausschnitt aus einem holistischen Digitalisierungs-KPI-Modell für produzierende Unternehmen dar, das vollständig in [9] beschrieben wird. In diesem Beitrag werden 36 der insgesamt 89 entwickelten $\mathrm{KPI}$ ausdifferenziert.

\section{Digitale Strategie und Führung geben Orientierung}

Die Entwicklung einer Vision, bzw. digitalen Strategie sowie die Orchestrierung des Wandels durch eine entsprechende Führung sind zwei zentrale Elemente der digitalen Transformation [10-12]. Sie bilden die Grundlage, um die Herausforderungen, die durch die Digitalisierung
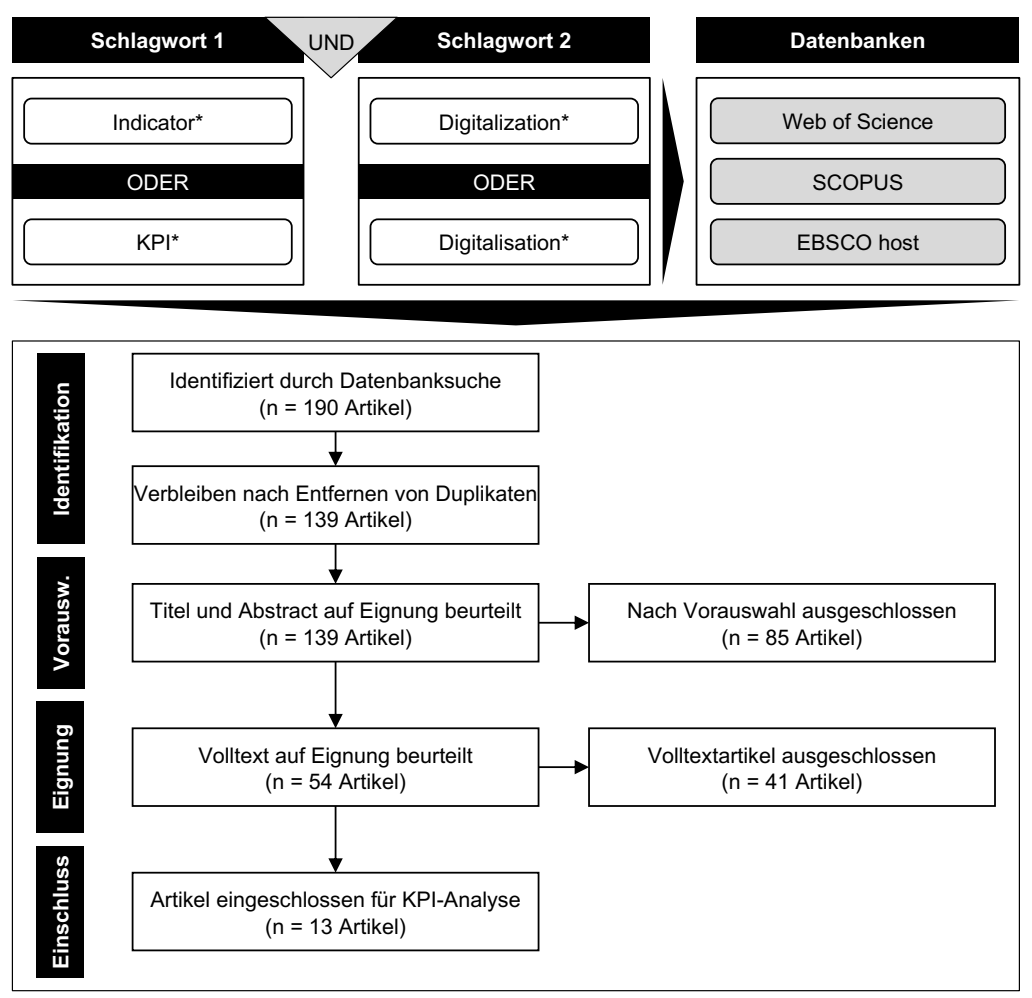

entstehen, strategisch bewältigen zu können [13] und schaffen damit auch die Basis für die Entwicklung und Implementierung neuer Geschäftsmodelle [14]. Entsprechende Faktoren sind demnach bei der Bewertung der digitalen Reife mittels KPI zu berücksichtigen. Als relevant identifizierte KPI sind in Bild 2 zu finden.

Mit dem Ziel, den disruptiven technologischen Veränderungen sowie daraus resultierendem Wandel im Käuferverhalten zu begegnen, verknüpft eine digitale Strategie die technologischen Informationssysteme mit den betrieblichen Managementstrategien zu einem adäquaten Geschäftsmodell [15-17]. Sie trägt zu einer nachhaltigen Leistungssteigerung und Sicherung der Wettbewerbsfähigkeit bei [13, 18]. Eine solche Strategie sollte demensprechend nicht nur vorhanden sein, sondern leicht verständlich und transparent für alle dokumentiert, regelmäßig aktualisiert und klar kommuniziert werden $[16,19]$.

Eine digitale Strategie bedarf einer adäquaten Umsetzung in Form digital geprägter Führungskonzepte. Bestehende Führungskonzepte sind dementsprechend dem digitalen Wandel anzupassen [20]. Dies geht mit der Forderung einher, dass sich das Management neuen Technologien widmet und eine Unternehmenskultur etabliert, in der Mitarbeiter motiviert werden, zur Ideenfindung und Innovations- sowie Organisationsentwicklung beizutragen $[16,21]$. Das Einnehmern einer Vorbild und Vorreiterrolle des Managements liefert einen wichtigen Beitrag
Bild 1: Suchstring,

Quellen und Flowchart der Systematischen Literaturrecherche. 
[1] Zimmermann, V: KfW-Digitalisierungsbericht Mittelstand 2019. Digitalisierungsprojekte zunehmend im Mittelstand verbreitet, Digitalisierungsausgaben jedoch seit Jahren unverändert niedrig. Frankfurt am Main 2020

[2] Kersten, W.; von See, B.; Lodemann, S.; Grotemeier, C. Trends und Strategien in Logistik und Supply Chain Management. Entwicklungen und Perspektiven einer nachhaltigen und digitalen Transformation. Bremen 2020.

[3] Brink, S.; Levering, B.; Icks, A Herausforderungen des deutschen Mittelstands in der $\mathrm{Co}$ rona-Pandemie. Sonderauswertung des Zukunftspanel Mittelstand 2020. Bonn 2020.

[4] Wrobel, M.; Schildhauer, T. Preiß, K.: Kooperation zwischen Startups und Mittel stand. Learn. Match. Partner. Berlin 2017.

[5] Nielen, S.; Kay, R.; Schröder, C.: Disruptive Innovationen: Chancen und Risiken für den Mittelstand. Bonn 2017.

[6] Leyh, C.; Bley, K.: Digitalisierung: Chance oder Risiko für den deutschen Mittelstand? - Eine Studie ausgewählter Unternehmen. In: HMD Praxis der Wirtschaftsinformatik 53 (2016) 1, S. 29-41.

[7] Franceschini, F: Galetto, $M$ : Maisano, D.; Neely, A. D.: Designing performance measurement systems. Theory and practice of key performance indicators. Cham 2019.

[8] Fink, A.: Conducting research literature reviews. From the internet to paper, Fourth edition. Thousand Oaks, California 2014.

[9] Krol, F.; Saeed, M. A.; Kersten, W.: A holistic digitalization KPI framework for the aerospace industry. In: Kersten, W: Blecker, T.: Ringle, C. M. (Hrsg.): Proceedings of the Hamburg International Conference of Logistics (HICL)/ Data Science and Innovation in Supply Chain Management. How Data Transforms the Value Chain. Berlin 2020.

[10] Kontić, L: Vidicki, Đ: Strategy for digital organization: Testing a measurement tool for digital transformation. In: Strategic Management 23 (2018) 2, S. 29-35.

[11] von See, B. Kersten, W.: Arbeiten im Zeitalter des Internets der Dinge. Wie Qualifikation, Organisation und Führung digital transformiert werden. In: Industrie 4.0 Management 34 (2018) 3, S. 8-12

[12] Porfírio, J. A.; Carrilho, T. Felício, J. A.: Jardim, J.: Leadership characteristics and digital transformation. In: Journal of Business Research 124 (2021), S. 610-19.

[13] Berghaus, S.; Back, A.; Kaltenrieder, B.: Digital Maturity \& Transformation Report 2017. St. Gallen 2017

in der Überzeugung der Mitarbeiter von der Notwendigkeit des digitalen Wandels [18, 21, 22].

\section{Digitalkompetenzen / Humankapital schaffen Basis}

Der Erfolg der digitalen Transformation eines Unternehmens hängt wesentlich von dessen Digitalkompetenzen bzw. dessen Humankapital ab [18, 23]. Neben spezifischen IT-Kenntnissen sind dabei jedoch auch sog. Fast Failure-Kompetenzen förderlich [24]. Eine entsprechende Messung des digitalen Reifegrads hat somit sowohl relevante Kenntnisse in der Informations- und Kommunikationstechnik (IKT) als auch Aspekte wie bspw. die Bereitschaft zum lebenslangen Lernen, die Offenheit für neue Technologien und das Vorhandensein interdisziplinären Denkvermögens zu umfassen [14, 18, 25-28]. Weiterhin sind sowohl bei der Personalentwicklung als auch -akquise spezifische Fort- und Weiterbildungsmaßnahmen und Gestaltungsfreiheiten der Mitarbeiter zu berücksichtigen [14, 16, 19, 23, 26]. Im Bereich der Digitalkompetenzen relevante KPI sind in Bild 3 zusammengefasst.

Digitalkompetenzen der Mitarbeiter umfassen unter anderem Informations- und Datenkompetenzen, die grundsätzlich für die Arbeit in einer digitalen Umgebung notwendig sind. Dies betrifft insbesondere Fähigkeiten zum Abruf und zur Analyse von digitalen Daten, beispielsweise durch das Internet, und die Nutzung verschiedener Endgeräte [29]. Gleichzeitig sollten Unternehmen qualifizierte Mitarbeiter aus dem IKT-Bereich beschäftigen, um die notwendige technische Unterstützung sicherzustellen.

Um in einer digitalen Arbeitsumgebung zu kollaborieren, benötigen Mitarbeiter Kompetenzen in der digitalen Kommunikation und Zusammenarbeit [13, 29, 30]. Dazu sollten die Mitarbeiter in der Lage sein, mittels digitaler Plattformen und digitaler Kommunikationstools sowie -medien zusammenzuarbeiten. Dies ermöglicht Unternehmen einerseits den Einsatz von flexiblen Arbeitsformen, andererseits können Mitarbeiter dadurch standortunabhängig Wissen und Ideen entlang der gesamten Wertschöpfungskette miteinander austauschen [13, 30, 31].

Die digitale Arbeitsumgebung stellt, verglichen mit der klassischen Arbeitsumgebung, andere Anforderungen an die Sicherheit und den Schutz der physischen und psychischen Gesundheit der Mitarbeiter [21, 29]. Mitarbeiter müssen die Auswirkungen digitaler Technologien auf das soziale Wohlbefinden verstehen [32] und auch im digitalen Kontext eine respektvolle, vielfältige und kollaborative Arbeitskultur etablieren [21].

Um in einer digitalen Arbeitswelt effizient arbeiten zu können, sollten Mitarbeiter auftretende Probleme selbstständig lösen können [29]. Dies umfasst nicht nur das technische (Fach-) Wissen, sondern setzt auch tiefergehendes Systemdenken und Prozessverständnis voraus [14]. Unternehmen sind daher gefordert, die Problemlösungsfähigkeiten ihrer Mitarbeiter systematisch durch regelmäßige Aus- und Weiterbildungen fördern und entwickeln [14].

Zur Integration digitaler Informationen in Geschäftsprozesse benötigen Mitarbeiter zudem die Kompetenz, digitale Inhalte und Informationen zu erstellen und zu bearbeiten [29]. Die Fähigkeit, aus Daten eine Wertschöpfung zu generieren (z. B. durch die Anwendung von Datenanalyse-Technologien), gewinnt dabei zunehmend an Relevanz [14, 23].

\section{Strategie und Führung}

\section{Digitale Strategie}

Digitale Strategie dokumentiert, regelmäßig aktualisiert und transparent kommuniziert

Roadmap/Strategieprozess für digitale Transformation ist Teil der Unternehmensstrategie

Umsetzungsstand der digitalen Strategie wird regelmäßig gemessen und verfolgt

Pers./finanz. Mittel sowie Einstellungsstrategie zur Umsetzung der Digital-Strategie vorhanden

Digitale Kompetenz ist Bestandteil der Personalentwicklungsstrategie

\section{Digitale Führung}

Fähigkeit vorhanden, Lücken im Bereich der Digitalkompetenz aufzudecken und zu schließen

Konsistente kanalübergreifende operative Führung mit externen Stakeholdern

Kontinuierliche Weiterentwicklung und Kommunikation der Führungskultur

Kontinuierliche Verbesserung des Managementsystems und dessen Performance

Mittleres Management entwickelt Mission/Vision/ Werte/Leitbild und agiert als Vorbild

Legende:

$=$ Qualitative Bewertung

= Quantitative/prozentuale Bewertung

Bild 2: KPI für das Themengebiet „Strategie und Führung“. 
Digitalkompetenzen/Humankapital

\section{Informations- und Datenkompetenz}

Mitarbeiteranteil, der Computer/Tablet/Smartphone am Arbeitsplatz nutzt

Mitarbeiteranteil, der Internet nutzt

Mitarbeiteranteil mit grundl. Digitalkompetenzen (z. B. Internetnutzung, Daten/Dateien bearbeiten)

- Mitarbeiteranteil im IKT-Sektor (inkl. Software, Hardware, Telekommunikation, verb. Dienstl.)

\section{Kommunikation und Zusammenarbeit}

- Mitarbeiteranteil, der mittels Digitaltechnologien zusammenarbeitet (z. B. Kollaborationsplattform)

- Mitarbeiteranteil, der digitale Kommunikations-

tools nutzt (z. B. Videoanrufe, soziale Netzwerke)

Mitarbeiteranteil, der das Internet zur

Kommunikation nutzt (z. B. Emails, Messenger)

\section{Sicherheit und Schutz}

IT-Sicherheitsvorschriften sind Mitarbeitern bekannt und werden regelmäßig evaluiert Regeln vorhanden, die Wohlbefinden/Gesundheit der Mitarbeiter gewährleisten

\section{Intelligente Prozesse schaffen Vernetzung}

Horizontale und vertikale Vernetzung der Lieferkette sind zwei zentrale Ziele einer digitalen Transformation [33, 34]. Intelligente Prozesse bilden eine zentrale Voraussetzung für ebendiese [14]. Mithilfe geeigneter KPI ist zu überprüfen, inwiefern die Prozesse unter Rücksichtnahme der bestehenden Systeme und Komponenten automatisiert, dezentralisiert und durchgängig gestaltet sind [13, 14, 21, 26]. In den Fokus sind dabei nicht nur die unternehmensinternen, sondern auch unternehmensübergreifenden Prozesse zu rücken und somit die Vernetzung entlang der gesamten Wertschöpfungskette vom Lieferanten bis zum Kunden zu forcieren [14]. Bild 4 liefert eine Zusammenfassung aller relevanten KPI, die der Beurteilung des Reifegrads intelligenter Prozesse dienen.

Um intelligente Prozesse zu etablieren, ist zunächst die Datenverarbeitung in der Produktion notwendig. Durch die Digitalisierung der physischen Produktionsausrüstung in der Fabrik kann die Verbindung zur virtuellen Welt hergestellt werden [14]. Die aus der Produktion generierten Daten können dadurch gesammelt, gespeichert und verarbeitet werden und stehen anschließend für die autonome Planung und Steuerung der Produktionsprozesse zur Verfügung $[14,35,36]$.

Damit den Mitarbeitern die produktionsrelevanten Informationen zur richtigen Zeit

\section{Problemlösefähigkeit}

Mitarbeiteranteil, der Online-Weiterbildungsangebote nutzt

$\checkmark$ Regelmäßige Weiterbildung der Digitalkompetenzen wird Mitarbeitern angeboten Mitarbeiteranteil, der industriespezifische Weiterbildung für digitale Anforderungen durchläuft

- Mitarbeiteranteil mit IKT-Problemlösungsfähigkeiten

\section{Digitale Inhaltserstellung}

- Mitarbeiteranteil, der Werkzeuge zum Analysieren und Entwickeln von digitalen Inhalten nutzt

- Mitarbeiteranteil mit speziellen Digitalkompetenzen (z. B. IKT-Spezialisten, Programmierer)

Mitarbeiteranteil, der aus Daten wertvolle

Erkenntnisse gewinnen kann

Bild 3: KPI für das Themengebiet „Digitalkompetenzen/Humankapital“.

am richtigen Ort zur Verfügung stehen, sind Mensch-Maschine-Schnittstellen in der Produktion erforderlich [35, 36]. Diese Schnittstellen werden beispielsweise durch mobile Endgeräte wie Tablets oder Smartphones sowie Datenbrillen bereitgestellt und können die Produktionseffizienz sowie betriebliche Abläufe erheblich verbessern [36].

Neben der Schnittstelle zwischen Mensch und Maschine hat auch die Kommunikation von Maschine zu Maschine eine Relevanz: Durch Datenschnittstellen in den Produktionsanlagen (bspw. Feldbus-, Ethernet- und Web-Schnittstellen) kann ein autonomer Informationsaustausch zwischen den Anlagen ermöglicht werden [35]. Dies begünstigt die standortunabhängige und unternehmensübergreifende Vernetzung der Produktion entlang der gesamten Wertschöpfungskette [35, 36].

Nicht nur innerhalb der Produktion, sondern auch zwischen Produktion und anderen Unternehmensfunktionen ist eine unternehmensweite Vernetzung notwendig [28, 35]. Dazu werden konsistente, einheitliche Dateiformate und harmonisierte IT-Lösungen benötigt, um die verschiedenen Unternehmensfunktionen (z. B. Beschaffung oder Vertrieb) mit der Produktion und den entsprechenden Produktionsdaten zu vernetzen $[33,34]$.

Voraussetzung für den Austausch von Produktionsdaten entlang der Wertschöpfungskette ist eine robuste Informations- und Telekommu-
[14] Lichtblau, K. Stich, V: Bertenrath, R.; Blum, M.; Bleider, M. Millac, A.; Schmitt, K.; Schmitz, E.; Schroeter, M.: Industrie 4.0 Readiness. Aachen, Cologne 2015.

[15] Waspodo, B.; Ratnawati, S. Halifi, R.: Building Digital Strategy Plan at CV Anugrah Prima an Information Technology Service Company: The 6th International Conference on Cyber and IT Service Management (CITSM 2018) 2018.

[16] Azhari, P. Faraby N.: Rossmann, A.; Steimel, B.; Wichman, K. S.: Digital Transformation Report. Köln 2014.

[17]Deloitte: Digital Maturity Model. Achieving digital maturity to drive growth. New York 2018

[18] BSP Business School Berlin: Mitelstand im Wandel - Wie ein Unternehmen seinen digitalen Reifegrad ermitteln kann. Berlin 2016.

19] KPMG: Digital Readiness Assessment. Berlin 2016.

[20] Buhse, W.: Management by Internet. Neue Führungsmodelle für Unternehmen in Zeiten der digitalen Transformation; Unternehmen im Wandel, digitale Medien als Werkzeugkoffer für Veränderer, Vernetzung, Offenheit, Partizipation und Agilität als Werte einer neuen Unternehmenskultur. Kulmbach 2014.

[21]EFQM: EFQM Excellence Modell. Brussels 2012

[22] Stowasser, S.: Peschl, A: Arbeitswelt im Wandel: Qualifizierung zu resilienzfördernder Führung. In: Spath, D.; Spanner-Ulmer, B. (Hrsg.): Digitale Transformation - Gutes Arbeiten und Qualifizierung aktiv gestalten. Berlin 2019.

[23] Geissbauer, R.; Vedso, J. Schrauf, S.: Industry 4.0: Building the digital enterprise 2016.

[24] Kersten, W.; Seiter, M.; See, B. von; Hackius, N.: Maurer, T. Chancen der digitalen Transformation. Trends und Strategien in Logistik und Supply Chain Management. Hamburg 2017.

[25] European Commission: The Digital Economy and Societyx Index (DESI). Integration of Digital Technology. URL: https://ec.europa.eu/ newsroom/dae/document. cfm?doc_id=59979. Abrufdatum 08.04.2020.

[26] Schumacher, A.; Erol, S.; Sihn W.: A Maturity Model for Assessing Industry 4.0 Readiness and Maturity of Manufacturing Enterprises. In: Procedia CIRP 52 (2016), S. 161-66.

[27] Kotarba, M.: Measuring Digitalization - Key Metrics. In Foundations of Management 9 (2017), S. 123-38.

[28] Dombrowski, U.; Wullbrandt J. Fochler, S.: Kompetenzentwicklung in der digitalen Transformation: dezentrales und lebenslanges Lernen im Arbeitsprozess. In: Spath, D Spanner-Ulmer, B. (Hrsg.): Digitale Transformation - Gutes Arbeiten und Qualifizierung aktiv gestalten. Berlin 2019. 
Bild 4: KPI für das Themengebiet „Intelligente Prozesse".
[29] Carretero, S.; Vuorikari, R.; Pu nie, Y.: DigComp 2.1. The digital competence framework for citizens with eight proficiency levels and examples of use. Luxembourg 2017.

[30] IBF Intranet Benchmarking Forum: Digital Workplace Maturity Model. London 2010

[31] Nabitz, U.; Klazinga, N.; Walburg, J.: The EFQM excellence model: European and Dutch experiences with the EFOM approach in health care. In International Journal for Quality in Health Care 12 (2000) 3, S. 191-202.

[32] Vuorikari, R., Punie, Y., Carretero Gomez S. \& Van den Brande, G.: DigComp 2.0: The Digital Competence Framework for Citizens, The Conceptual Reference Model. ceptual Reference

[33] Bischoff, J.; Taphorn, C.; Wolter, D.: Braun, N.; Fellbaum, M. Goloverov, A: Ludwig, S: Hegmanns, T.; Prasse, C.; Henke, M.; ten Hompel, M.; Döbbele F.; Fuss, E.; Kirsch, C.; Mättig, B Braun, S.; Guth, M.; Kaspers, M. Scheffler, D. Erschließen der Potenziale der Anwendung von Industrie $4.0 \mathrm{im}$ Mittelstand. Studie. Mülheim an der Ruhr 2015.

[34] Kagermann, $H_{\text {. }}$ Wahlster W.; Helbig, J.: Umsetzungsempfehlungen für das Zukunftsprojekt Industrie 4.0 München 2013.

[35]VDMA: Guideline Industrie 4.0. Guiding principles for the implementation of industrie 4.0 in small and medium sized businesses. Frankfurt am Main 2016.

[36] IHK München und Oberbayern: Selbstcheck - Industrie 4.0. URL: https://ihk-industrie40.de/selbstcheck/. Abrufdatum 18.05.2020.

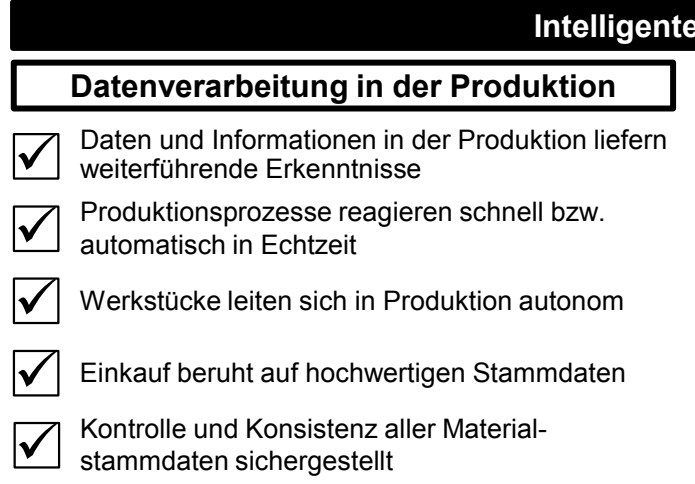

$\checkmark$ Mensch-Maschine-Schnittstellen

Mensch-Maschine-Schnittstellen in der Produktionsumgebung vorhanden

nikationsinfrastruktur in der Produktion [35, 36]. Nur bei Vorhandensein der entsprechenden Infrastruktur können die benötigten (Produktions-) Daten zeitnah oder in Echtzeit erfasst und für technische und organisatorische Prozessverbesserungen genutzt werden [35].

Abschließend führt die Kundenanforderung nach hochindividualisierten Waren und Produkten zu zunehmend kleineren Losgrößen (bis hin zur Losgröße 1). Dadurch steigt die Komplexität der Produktionsprozesse, insbesondere auch für produzierende und hochspezialisierte KMU [35]. Um auch bei kleinen Chargen mit hoher Effizienz fertigen zu können, sollten die Produktionsprozesse flexibel und modular strukturiert und eng mit der Auftragsplanung und -abwicklung verzahnt werden [34].

\section{Fazit und Ausblick}

In der aktuellen Krisenphase ist eine zielgerichtete Allokation von Digitalisierungs-Investitionen unabdingbar. Eine erfolgreiche digitale Transformation bedarf einer kontinuierlichen Überwachung und Weiterentwicklung. Hierbei ist der Fokus jedoch nicht nur auf technologische Aspekte zu richten, sondern das soziotechnische System in seiner Gesamtheit zu berücksichtigen. KPI bieten Managern die Möglichkeit, den aktuellen Fortschritt bei der digitalen Transformation zu messen und entsprechend Investitionen zu budgetieren. Bild 2 bis 4 liefert eine Zusammenfassung relevanter KPI mit Fokus auf die Mensch-Technik-Interaktion.

Zusammenfassend erweisen sich drei zentrale Themenfelder von Relevanz zur Überprüfung des Fortschritts bei der digitalen Transformation mithilfe von dezidierten KPI: erstens der Umsetzungsstand einer digitalen Vision bzw. digi- talen Strategie sowie die Orchestrierung des Wandels durch eine entsprechende Führung, zweitens die im Unternehmen vorhandenen IT-Kenntnisse, aber auch umsetzungsrelevanten Sozial- und Persönlichkeitskompetenzen sowie drittens die unternehmensinterne sowie unternehmensübergreifende intelligente Prozessgestaltung.

Die unter diesen Themenfeldern identifizierten KPI weisen unterschiedliche Aggregationslevel auf und bedürfen insbesondere bei den qualitativ zu bewertenden KPI (Bild 2-4) einer weiteren Spezifizierung im Unternehmen. Bei der Operationalisierung ist dabei unternehmensindividuell festzulegen, welche Ausprägung des jeweiligen KPI als Optimum angesehen wird. So muss bspw. der Mitarbeiteranteil, der aus Daten wertvolle Erkenntnisse gewinnen kann (Bild 3) nicht zwingend $100 \%$ sein, sondern ist entsprechend der Digitalstrategie zu definieren. Auf diese Weise kann das hier vorgestellte KPI-Modell zukünftig als Grundlage für die unternehmensindividuelle Bestimmung des digitalen Reifegrads und darauf aufbauend für die in diesen Krisenzeiten so wichtige Allokation von Digitalisierungs-Investitionen genutzt werden.

Dieser Beitrag entstand im Rahmen des Projekts "Digitalization and Internationalization Maturity Level in Aerospace (DIMLA)", das vom Bundesministerium für Bildung und Forschung und vom Projektträger Jülich unter dem Förderkennzeichen 03INT504CB gefördert wird.

\section{Schlüsselwörter:}

Digitale Transformation, Kennzahlen, Soziotechnisches System, Industrie 4.0 